\title{
interview
}

\section{Backwards torque}

\section{Etienne Brasselet explains to Nature Photonics that light can exert a counter-intuitive torque on matter.}

What is a left-handed mechanical effect?

Physicists sometimes refer to a phenomenon as being 'left handed' to emphasize its counter-intuitive nature. Common examples are the unusual wave propagation properties (such as negative refraction) of some man-made materials; these properties and materials are often referred to as being left handed. By analogy, a left-handed mechanical effect involves the manifestation of a force (or torque) exerted on a material system that is counter-intuitive in terms of the linear (or angular) momentum that produces it. As an illustration, when you catch a ball, your hand experiences a force that pushes it in the direction the ball is moving (that is, parallel to the linear momentum of the ball). If the ball is spinning, you will also experience a torque that tends to twist your hand with the same handedness as that of the spinning motion of the ball; in other words, the torque will have the same sign as the angular momentum of the ball. Left-handed versions of these mechanical effects would correspond to the incoming ball pulling your hand in the opposite direction to the ball's motion and twisting your hand in the opposite direction to the angular momentum of the ball - a rather surprising experience!

How is your work related to 'negative optical forces', and what has been done previously in the context of left-handed mechanical effects?

Negative optical forces refer to forces that push objects upstream of an incident photon flux. This actually represents a left-handed optomechanical manifestation associated with the linear momentum of light. As far as we are aware, only lefthanded forces induced by waves have been observed to date. These observations are mainly experimental demonstrations involving light fields, although acoustic fields have also been used recently. In addition to force, torque is another mechanical degrees of freedom that can manipulate matter. It was therefore tempting to consider the angular analogue of negative optical forces. This corresponds to a light field exerting a radiation torque on matter, where the direction of the torque is opposite to that of the incident angular

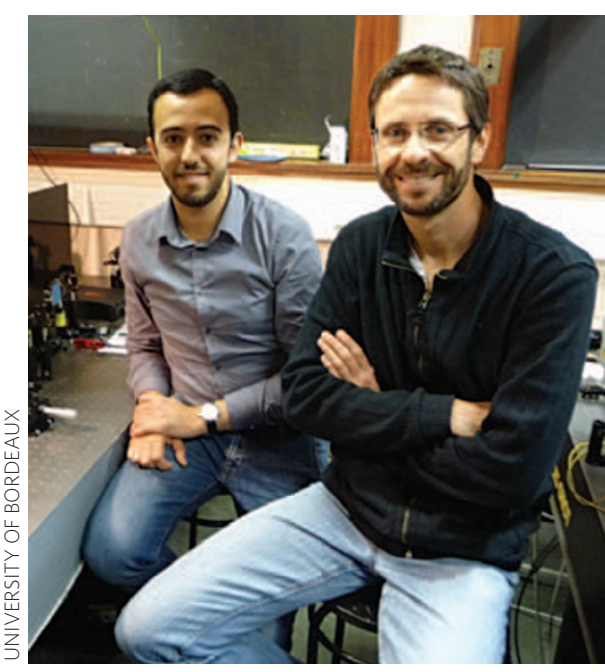

Etienne Brasselet (right) and Davit Hakobyan at Laboratoire Ondes et Matière d'Aquitaine, University of Bordeaux, France, have demonstrated left-handed optical radiation torque.

momentum. Such a generalization has motivated the description of mechanical effects as being 'left-handed' rather than 'negative', as this terminology avoids potential confusion with the sign of the incident angular momentum, which can be positive or negative, irrespective of whether it induces expected or counter-intuitive mechanical effects.

\section{What have you demonstrated in your present work?}

We have extended the concept of lefthanded optical forces to optical torques and provided an experimental demonstration of it. Practically, we have shown that a circularly polarized Gaussian beam has the ability to rotate objects in the opposite direction to that of the spin angular momentum carried by the incident photons.

\section{What was the main challenge?}

This work consisted of two main steps. The first was to identify a situation that would enable the observation of a left-handed optical radiation torque. Left-handed optical forces are basically associated with the net forward scattering of the linear momentum of light. By analogy, the challenge was to produce a kind of 'forward scattering' of the angular momentum of light, irrespective of the sign of the driving incident angular momentum. Considering an incident circularly polarized Gaussian beam, the idea was to couple the incident spin angular momentum to the spatial degrees of freedom of the light field, namely the orbital angular momentum. Indeed, if the optical torque is dominated by the orbital contribution rather than that of the spin, it becomes possible to reverse the angular momentum balance. We thus chose a material system that exhibits spin-orbit light scattering and deliberately fabricated optically inhomogeneous and anisotropic transparent objects.

The second step was to detect experimentally the mechanical effect of tiny optical torques, which typically are of the order or less than $10^{-15} \mathrm{~N} \mathrm{~m}$ for $1 \mathrm{~W}$ illumination of our centimetre-sized objects. Indeed, it was too difficult to observe reverse light-induced rotation directly. This difficulty was circumvented by probing the mechanical effect of the optical torque on the light itself in a rotational Doppler experiment. Using the principle of energy conservation, we can determine the optical torque exerted on an object from the measured rotational Doppler frequency shift of light interacting with the object in controlled motion about the propagation axis of the incident beam. Such a frequency shift is measured with a high precision by interferometric techniques.

Are the immediate implications mainly academic for now?

Our primary aim was to equip the left-handed optomechanical toolbox with angular features. By proposing a general framework to achieve it and reporting its experimental demonstration, this work should promote the use of unconventional mechanical effects of waves to manipulate matter. Although emerging left-handed mechanical effects may find applications, further fundamental developments may unravel new counter-intuitive aspects of wavematter interaction.

\section{INTERVIEW BY DAVID PILE}

Etienne Brasselet and Davit Hakobyan have a Letter on left-handed optical radiation torque on page 610 of this issue. 